\title{
Lock-exchange flows in inclined pipes: the relevance of the Prandtl mixing length model
}

\author{
Yukie Tanino [1 *, Frédéric Moisy and Jean-Pierre Hulin \\ Université Paris-Sud, CNRS., Laboratoire FAST, Bât. 502, Campus Universitaire, Orsay, France
}

(Received 20 June 2014; accepted 26 December 2014)

\begin{abstract}
We examine the applicability of the Prandtl mixing length model to transverse momentum and mass flux in strongly confined, stably stratified turbulent shear flows. These fluxes were measured in the vertical diametral plane of lock-exchange flows in an inclined pipe by the simultaneous use of planar laser-induced fluorescence and particle image velocimetry at local Reynolds numbers ranging from $\mathrm{Re}=580$ to 1770 and Richardson numbers ranging from $\mathrm{Ri}=0.26$ and 1.6. Measurements indicate that the eddy diffusivities of mass and momentum are symmetric about the pipe axis, with their maximum at the axis. The corresponding Prandtl mixing lengths decrease with increasing distance from the pipe axis within the central $60 \%$ of the pipe cross-section. Within the range of experimental conditions, the mixing lengths at the axis increase linearly with Ri so that the corresponding turbulent Prandtl number $\operatorname{Pr}_{t}$ decreases with $\mathrm{Ri}$. In contrast, $\mathrm{Pr}_{\mathrm{t}}$ and the mixing lengths do not display a systematic dependence on Re. Comparison with unbounded and semi-bound shear flows suggests that the strong confinement imposed by the pipe wall may be constraining the integral length scale and Prandtl mixing lengths.
\end{abstract}

Keywords: Prandtl mixing length; stratified shear flow; eddy diffusivity

\section{Introduction}

Turbulent transverse fluxes of momentum, heat, and scalar constituents have been studied for decades because of their relevance to a wide range of industrial and environmental processes, e.g., well cementing in oil and gas reservoirs,[1] thermal stimulation of hydrate reservoirs for gas production,[2] suspension feeding and the corresponding phytoplankton distribution in coastal waters,[3-5] air quality in residential areas,[6] and heat and vapour flux between trees and the overlying atmosphere.[7,8] In particular, with forests constituting about $30 \%$ of the earth's land surface,[9] fluxes from these forests can play a significant role in determining the global climate. $[9,10]$

In many flows, transverse fluxes are characterised by the classic gradient-flux model, which describes the flux as a product of the transverse gradient of the mean of the variable of interest (e.g., velocity) and an eddy diffusivity. The most common approach for predicting the eddy diffusivity is the Prandtl mixing length model.[11-19] One criterion for eddy diffusivities to be constant in space, as is the case with molecular diffusion, is for the mean gradient to vary at scales much larger than those of the mechanisms contributing to the mixing.[20] This condition is generally not met near a solid boundary, and it is now well

*Corresponding author. Email: ytanino@abdn.ac.uk 
established that eddy diffusivities vary strongly within many wall-bounded flows. Mixing lengths in such flows similarly vary in the transverse direction.

In this paper, we consider turbulent fluxes of momentum and concentration of a solute in a stratified shear flow in an inclined pipe generated as a lock exchange: denser and lighter fluids occupying the upper and lower halves of the pipe, respectively, are placed into contact at time $t=0$. The axial density gradient drives an exchange flow,[21] whereby the heavier fluid propagates downward along the lower portion of the pipe cross-section and the lighter fluid propagates along the upper portion in the opposite direction. Because of the geometry of the system, flow properties vary strongly in the transverse direction. The solute is initially uniformly distributed within each fluid, at concentration $C_{1}$ in the lighter fluid and concentration $C_{2}$ in the denser fluid.

We focus specifically on the stratified shear flow established far downstream of the propagating fronts, where turbulence is homogeneous in the streamwise direction and quasistationary. Efforts to date have focused on the flow structure and momentum transport in these flows [16,22] and the evolution of the mean velocity and density under intermittently turbulent conditions.[23] The focus of this paper is on the direct measurement of turbulent mass flux by the simultaneous use of planar laser-induced fluorescence (LIF) and particle image velocimetry (PIV). Transverse profiles of the mean velocity and concentration are presented in Section 3.1. The validity of the constant eddy diffusivity model and of the constant Prandtl mixing length model is evaluated in Sections 3.2 and 3.3, respectively. The dependence of the turbulent Prandtl number, the mixing lengths, the integral length scale of turbulence, and the turbulence intensity on the Richardson and Reynolds numbers are explored in Section 3.4.

\subsection{Gradient-flux model and the Prandtl mixing length}

The flow configuration of interest is illustrated in Figure 1. The Cartesian coordinates $\mathbf{x}=$ $(x, y, z) \equiv\left(x_{1}, x_{2}, x_{3}\right)$ are defined so that the $x$-axis coincides with the axis of the pipe, the $z$-axis is in the vertical plane containing the axis of the pipe, and $x=0$ is at the partition (lock). The components of the fluid velocity, $\mathbf{u}(\mathbf{x}, t)=(u, v, w) \equiv\left(u_{1}, u_{2}, u_{3}\right)$, are aligned with the axes $(x, y, z)$, respectively.

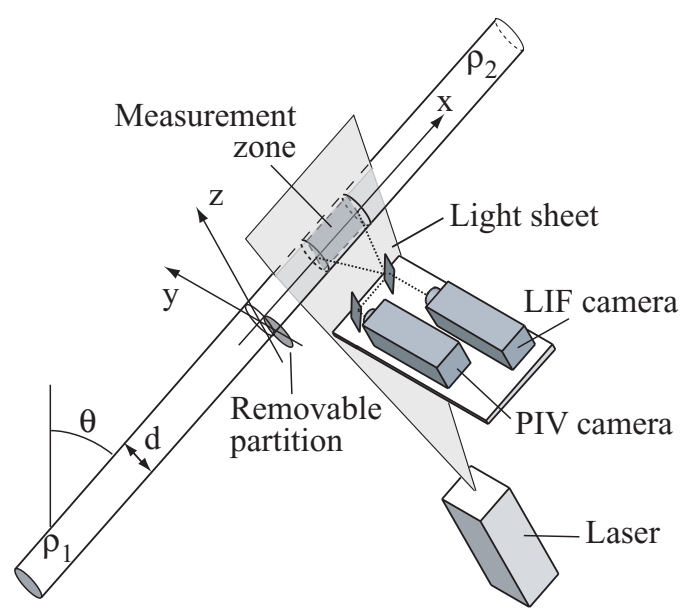

Figure 1. Central section of the experimental set-up. Not to scale. Figure redrawn from Ref. [23]. 
This paper is focused on uniformly turbulent, fully developed flow established far downstream of the propagating fronts, i.e., the flow does not display slow variations in time or in $x$. Moreover, only the vertical diametral plane $(y=0)$ is considered. To first order, within $|y| \lesssim 25 \%$ of the pipe diameter and far from the wall,[16] the time-averaged velocity, $\bar{u}_{i}$, varies predominantly in $z$. The overbar denotes a temporal averaging operation over an interval much longer than the scale of turbulent fluctuations, but within the duration over which the flow remains quasi-steady and the turbulence, stationary. Because the dominant variation is along $z$, it is convenient to further average variables over $x$. This spatial averaging operation, denoted by \langle\rangle$_{x}$, is performed within a volume interval that extends over a streamwise length much longer than the integral length scale of turbulence but much shorter than the distance to the propagating front. With these definitions, the turbulent fluctuations in velocity are then given by $u_{i}^{\prime}(x, z, t)=u_{i}(x, z, t)-\left\langle\overline{u_{i}}\right\rangle_{x}(z)$. By definition, $\left\langle\overline{u_{i}^{\prime}}\right\rangle_{x}=0$.

The common approach to turbulence closure for such flows is to use the gradient-flux model, which expresses the turbulent fluxes of momentum and scalar in the transverse direction as

$$
\left\langle\overline{w^{\prime} u^{\prime}}\right\rangle_{x}=-\epsilon_{\mathrm{m}} \frac{\mathrm{d}\langle\bar{u}\rangle_{x}}{\mathrm{~d} z}
$$

and

$$
\left\langle\overline{w^{\prime} c^{\prime}}\right\rangle_{x}=-\epsilon_{c} \frac{\mathrm{d}\langle\bar{c}\rangle_{x}}{\mathrm{~d} z}
$$

respectively, where $\epsilon_{\mathrm{m}}$ and $\epsilon_{c}$ are known as eddy diffusivities of momentum and mass, $c(x, z, t)$ is the mass concentration of a constituent species, and

$$
c^{\prime}(x, z, t)=c(x, z, t)-\langle\bar{c}\rangle_{x}(z)
$$

is its turbulent fluctuation.

The Prandtl mixing length model further relates $\epsilon_{\mathrm{m}}$ and $\epsilon_{c}$ to the mean velocity gradient as

$$
\epsilon_{\mathrm{m}}=l_{\mathrm{m}}^{2}\left|\frac{\mathrm{d}\langle\bar{u}\rangle_{x}}{\mathrm{~d} z}\right|
$$

and

$$
\epsilon_{c}=l_{c} l_{\mathrm{m}}\left|\frac{\mathrm{d}\langle\bar{u}\rangle_{x}}{\mathrm{~d} z}\right|,
$$

where $l_{\mathrm{m}}\left|\partial\langle\bar{u}\rangle_{x} / \partial z\right|$ is the characteristic velocity of the turbulent eddies and $l_{\mathrm{m}}$ and $l_{c}$ are characteristic mixing lengths for momentum and mass, respectively.

\section{Materials and methods}

Laboratory experiments were performed to measure concentration and velocity simultaneously to determine the eddy diffusivities of momentum and mass, $\left(\epsilon_{\mathrm{m}}, \epsilon_{c}\right)$ (Equation (1)), and the corresponding Prandtl mixing lengths, $\left(l_{\mathrm{m}}, l_{c}\right)$ (Equation (3)). 


\subsection{Experimental set-up and procedure}

Lock-exchange flows were generated in a $d=20 \mathrm{~mm}$-diameter plexiglas pipe consisting of two sections of length $L=1670 \mathrm{~mm}$ separated by a transverse partition in the plane $x=0$ that can be opened or closed. The pipe was oriented at $\theta=15^{\circ}$ or $45^{\circ}$ to the vertical (Figure 1).

The instrumentation is identical to that described in Ref. [23], and the laboratory procedure largely the same, so only key features and steps are presented here. Prior to each experiment, the partition was closed. The lower pipe was filled with an aqueous solution of rhodamine $6 \mathrm{G}$ of concentration $C_{1}=(161 \pm 2) \times 10^{-3} \mathrm{~g} \mathrm{~m}^{-3}$ or $(179 \pm 2) \times 10^{-3} \mathrm{~g} \mathrm{~m}^{-3}$ and density $\rho_{1}$. The upper pipe was filled with an aqueous solution of calcium chloride and rhodamine $6 \mathrm{G}$ of density $\rho_{2}\left(>\rho_{1}\right)$ and rhodamine concentration $C_{2}$ ranging from $(20.1$ $\pm 0.3) \times 10^{-3} \mathrm{~g} \mathrm{~m}^{-3}$ to $(22.4 \pm 0.3) \times 10^{-3} \mathrm{~g} \mathrm{~m}^{-3}$. Rhodamine $6 \mathrm{G}$ was added to both fluids to ensure that unintended residual rhodamine in the system would not interfere with the measurements. The value of $C_{1}$ was selected to make maximum use of the dynamic range of the 12-bit camera sensor that recorded the concentration. $\rho_{1}$ ranged from 997.8 to $999.0 \mathrm{~kg} \mathrm{~m}^{-3}$ and $\rho_{2}$ ranged from 1005.2 to $1026.3 \mathrm{~kg} \mathrm{~m}^{-3}$. Both liquids were seeded with glass spheres for PIV.

An experiment began with the removal of the partition at $t=0$. The propagation of the exchange flow was visualised using a pulsed laser sheet of wave length $\lambda=532 \mathrm{~nm}$ that illuminated the vertical, $y=0$ plane (Figure 1 ). In this plane, the pipe walls are at $\widetilde{z} \equiv z / d= \pm 1 / 2$; in this paper, $\sim$ denotes normalisation by $d$. The laser sheet was approximately $2 \mathrm{~mm}$ thick. Optical distortion from the curvature of the pipe was minimised by encasing the pipe in the region of interest in a transparent, square cell oriented so that two sides were parallel to the laser plane and filling the space between the pipe and the cell with water. The refractive indices of plexiglas and water under experimental conditions are $n \approx 1.495$ [24] and $n \approx 1.335$, [25] respectively. This difference in the refractive indices was previously found to have negligible impact on the local concentration measurements. [26] A long-pass dichroic mirror was used to separate the LIF of rhodamine $6 \mathrm{G}$ (peak $\lambda=555 \mathrm{~nm}$ ) from the incoming laser light and the reflection of the light by the seeding material (Figure 2). One CCD camera captured the LIF of rhodamine $6 \mathrm{G}$ and a second CCD camera imaged the seeding particles. The two cameras were positioned so that their fields of view coincided to within 3 pixels within the $65 \mathrm{~mm} \times 20 \mathrm{~mm}$ rectangular region of interest centered at

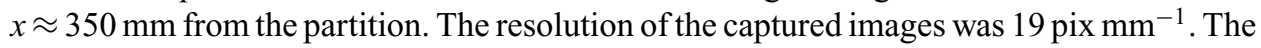
two cameras captured images simultaneously at 3.9 frames per s. The local longitudinal and transverse velocity components, $u$ and $w$, were determined by PIV using a final interrogation

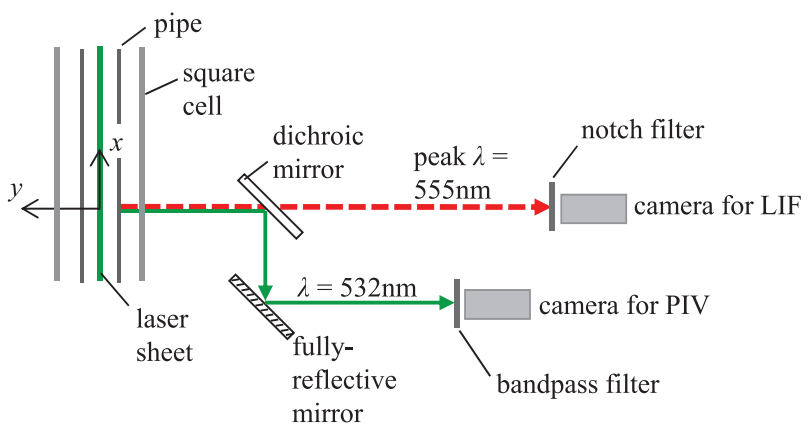

Figure 2. Optical set-up. Not to scale. 
window size of 16 pix $\times 16$ pix (i.e., $0.04 d \times 0.04 d$ ) with a $50 \%$ overlap between adjacent windows. The instantaneous concentration of rhodamine $6 \mathrm{G}, C(x, z, t)$, was determined using a monotonic polynomial calibration curve fitted to the LIF intensities recorded for known concentrations in the range $C_{2} \leq C \leq C_{1}$ as integer numbers on a scale between 0 and 4095.

As stated in Section 1, this paper is focused on quasi-steady flow established far downstream of the propagating fronts. Accordingly, we do not consider times $t<2 t_{\mathrm{f}}$, where $t_{\mathrm{f}}$ is the arrival time of the propagating front at the imaging region as identified from the recorded LIF, and $t>L / v_{\mathrm{f}}$, the approximate time of arrival of the front at the end of the pipe. The duration of each experiment, which corresponds to the interval $2 t_{\mathrm{f}}<t<L / v_{\mathrm{f}}$, varied between 83 and $189 \mathrm{~s}$.

\subsection{Reynolds decomposition}

Because the imaging window is offset to one side of the partition, the mean concentration within it increases slowly with $t$. This drift is in sharp contrast to $u$ and $w$, which do not display slow variations in $x$ or $t$ (e.g., Figure 3; also see [22,23]). Seon et al. [27] previously observed that the evolution of the cross-sectionally averaged concentration with $t$ and in $x$ in inclined lock-exchange flows may be described by the classic solution for 1-D diffusion. Following this observation, an error function of the form

$$
c_{\text {erf }}(x, t)=\frac{C_{1}-C_{2}}{2}\left[1-\operatorname{erf}\left\{\frac{x}{\sqrt{4 D_{x}\left(t-t_{0}\right)}}\right\}\right]
$$

was fitted, in the least squares sense, to $\langle C\rangle_{|\widetilde{z}|<0.25}-C_{2}$ measured over $2 t_{\mathrm{f}}<t<L / v_{\mathrm{f}}$, where $\langle C\rangle_{|\widetilde{z}|<0.25}$ denotes the average of $C$ over $|\widetilde{z}|<0.25 ; D_{x}$ and $t_{0}$ are fitting parameters (e.g., Figure 3). $c(x, z, t)=C(x, z, t)-C_{2}-c_{\text {erf }}(x, t)$ then represents the local, instantaneous concentration field without the underlying global trend.

Finally, to calculate the cross-correlation between concentration and velocity, $c(x, z, t)$ was linearly interpolated to the centres of the 16 pix $\times 16$ pix PIV interrogation cells used to compute $(u, w)$.

A single transverse profile of $\langle\bar{u}\rangle_{x}(z),\langle\bar{w}\rangle_{x}(z)$, and $\langle\bar{c}\rangle_{x}(z)$ was calculated for each experiment using $2 t_{\mathrm{f}}<t<L / V_{\mathrm{f}}$ as the interval for the time averaging operation and the streamwise span of the imaging region as the spatial $(x)$ averaging interval. The latter is $3.2 d$ long for all experiments, which is an order of magnitude longer than the integral length scale (Section 3.3). The time interval is also much longer than the time scale of the turbulent fluctuations (Section 2.1).

\subsection{Experimental conditions}

Nine experimental runs, classified into three pairs of values of the angle $\theta$ and the Atwood number $\left[\right.$ At $\left.=\left(\rho_{2}-\rho_{1}\right) /\left(\rho_{1}+\rho_{2}\right)\right]$, are considered in this paper (Table 1). Specifically, the $\left(\theta\right.$, At) pairs considered are $\left(15^{\circ}, 1 \times 10^{-2}\right),\left(15^{\circ}, 4 \times 10^{-3}\right)$, and $\left(45^{\circ}, 1.19 \times 10^{-2}\right)$. These combinations correspond to inertial velocity scales ranging from $V_{\mathrm{t}}=\sqrt{(g \cos \theta \operatorname{At} d) / 2}=$ 1.9 to $3.6 \mathrm{~cm} \mathrm{~s}^{-1}$ (cf. [21]). ${ }^{1}$ At $\left(\theta\right.$, At) $=\left(45^{\circ}, 4 \times 10^{-3}\right)$, the flow was found to display distinct, brief periods of low turbulence intensity,[23] and is thus excluded from the present analysis. The Schmidt number remained approximately constant at $\mathrm{Sc}=v / D_{\mathrm{m}} \approx$ 3400 to 3500 , where the molecular diffusion coefficient of rhodamine $6 \mathrm{G}$ is taken to be 
(a) $\left(15^{\circ}, 3.89 \times 10^{-3}\right)$
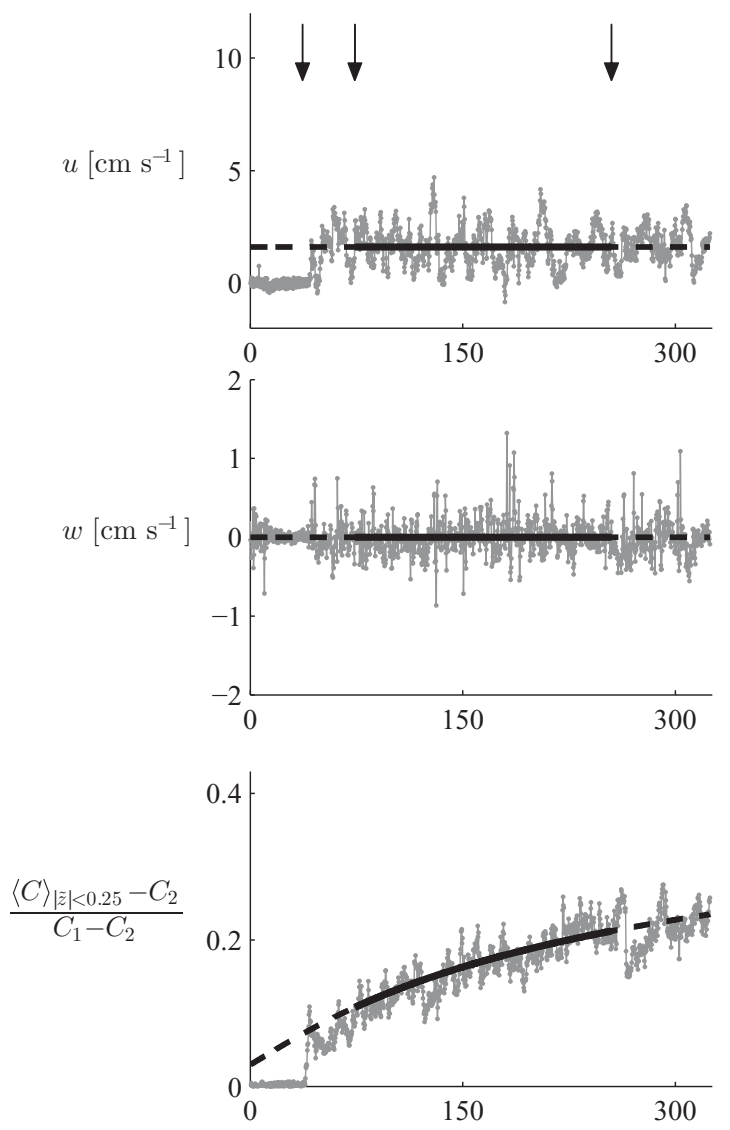

(b) $\left(45^{\circ}, 1.19 \times 10^{-2}\right)$
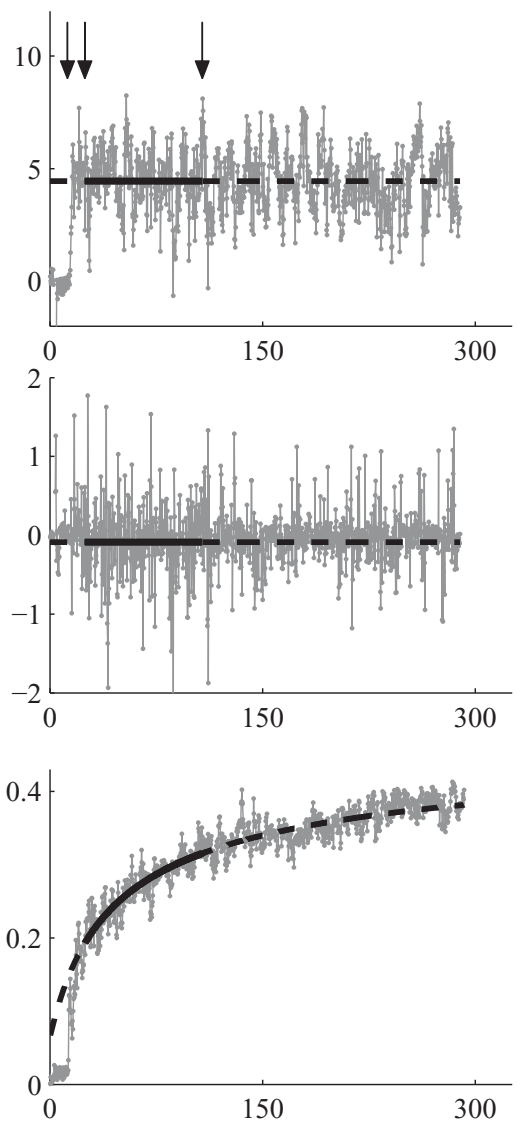

$t[\mathrm{~s}]$

Figure 3. The evolution of $u$ at selected $(x, z)$ (top), $w$ at the same $(x, z)$ (middle), and $\left(\langle C\rangle_{|z|} \mid<0.25-C_{2}\right) /\left(C_{1}-C_{2}\right)$ at selected $x$ (bottom) in runs J07 (left) and S21 (right). The thick solid lines correspond to $\bar{u}$ (top), $\bar{w}$ (middle), and a least squares fit of Equation (4) to data (bottom); the dashed lines are their extrapolation out with the averaging interval $2 t_{\mathrm{f}}<t<L / V_{\mathrm{f}}$. Arrows in the top subplots depict $t=t_{\mathrm{f}}, 2 t_{\mathrm{f}}$, and $L / V_{\mathrm{f}}$.

$D_{\mathrm{m}}=3 \times 10^{-6} \mathrm{~cm}^{2} \mathrm{~s}^{-1}[30]$ and the kinematic viscosity, $v$, is interpolated from values reported for aqueous calcium chloride at a reference temperature of $20^{\circ}$.[31] Note that $v$ corresponding to concentrations considered presently deviate at most by $5 \%$ from that of pure water at the same temperature $\left(v=1.003 \times 10^{-6} \mathrm{~m}^{2} \mathrm{~s}^{-1}\right)$. [32] The local Reynolds number, $\operatorname{Re}=\Delta U d / \nu$, calculated using

$$
\Delta U=\langle\bar{u}\rangle_{x}\left(\widetilde{z}^{+}\right)-\langle\bar{u}\rangle_{x}\left(\widetilde{z}^{-}\right),
$$

where $\widetilde{z}=\widetilde{z}^{-}$and $\widetilde{z}^{+}$are the transverse coordinates corresponding to minimum and maximum $\langle\bar{u}\rangle_{x}$, respectively, ranged from $\operatorname{Re}=580$ to 1770 (Table 1). Similarly, the Richardson 
Table 1. Experimental conditions. $V_{\mathrm{f}}$ is the equilibrium (long-time) front velocity as interpolated from measurements reported in Refs [28] (for At $=O(4) \times 10^{-3}$ ) and [29] (for At $=O(1) \times 10^{-2}$ ). The local Reynolds number, Re, is given by Equation (5). The Richardson number, Ri, is defined by Equation (6).

\begin{tabular}{|c|c|c|c|c|c|c|}
\hline Run & $\theta$ & At & $V_{\mathrm{t}}\left[\mathrm{cm} \mathrm{s}^{-1}\right]$ & $V_{\mathrm{f}}\left[\mathrm{cm} \mathrm{s}^{-1}\right]$ & $\operatorname{Re}$ & $\mathrm{Ri}$ \\
\hline $\begin{array}{l}\mathrm{J} 14 \\
\mathrm{~J} 19 \\
\mathrm{~J} 22\end{array}$ & $15^{\circ}$ & $\begin{array}{l}1.38 \times 10^{-2} \\
1.14 \times 10^{-2} \\
1.14 \times 10^{-2}\end{array}$ & $\begin{array}{l}3.61 \\
3.29 \\
3.28\end{array}$ & 0.74 & $\begin{array}{l}703 \\
724 \\
734\end{array}$ & $\begin{array}{l}1.59 \\
1.42 \\
1.25\end{array}$ \\
\hline $\begin{array}{l}\mathrm{J} 07 \\
\mathrm{~J} 05 \\
\mathrm{~J} 29 \\
\mathrm{~J} 02\end{array}$ & $15^{\circ}$ & $\begin{array}{l}3.89 \times 10^{-3} \\
3.84 \times 10^{-3} \\
3.94 \times 10^{-3} \\
3.69 \times 10^{-3}\end{array}$ & $\begin{array}{l}1.92 \\
1.91 \\
1.93 \\
1.87\end{array}$ & 0.65 & $\begin{array}{l}577 \\
582 \\
604 \\
605\end{array}$ & $\begin{array}{l}0.65 \\
0.63 \\
0.67 \\
0.54\end{array}$ \\
\hline $\begin{array}{l}\mathrm{S} 17^{\mathrm{a}} \\
\mathrm{S} 21^{\mathrm{a}}\end{array}$ & $45^{\circ}$ & $\begin{array}{l}1.19 \times 10^{-2} \\
1.19 \times 10^{-2}\end{array}$ & $\begin{array}{l}2.87 \\
2.87\end{array}$ & 1.55 & $\begin{array}{l}1770 \\
1770\end{array}$ & $\begin{array}{l}0.27 \\
0.26\end{array}$ \\
\hline $\mathrm{T} 3^{\mathrm{b}}$ & $15^{\circ}$ & $1.15 \times 10^{-2}$ & 3.30 & 0.74 & $\begin{array}{l}770 \\
780 \\
640 \\
670\end{array}$ & $\begin{array}{l}1.2 \\
1.2 \\
1.8 \\
1.7\end{array}$ \\
\hline $\mathrm{T} 1^{\mathrm{b}}$ & $15^{\circ}$ & $4.3 \times 10^{-3}$ & 2.02 & 0.65 & $\begin{array}{l}680 \\
650 \\
640\end{array}$ & $\begin{array}{l}0.55 \\
0.58 \\
0.53\end{array}$ \\
\hline $\mathrm{T} 4^{\mathrm{b}}$ & $30^{\circ}$ & $1.48 \times 10^{-2}$ & 3.55 & 1.14 & $\begin{array}{l}1260 \\
1280 \\
1280\end{array}$ & $\begin{array}{l}0.69 \\
0.73 \\
0.65\end{array}$ \\
\hline $\mathrm{T} 2^{\mathrm{b}}$ & $30^{\circ}$ & $4.1 \times 10^{-3}$ & 1.86 & 0.93 & $\begin{array}{l}1060 \\
1030 \\
1000 \\
1000\end{array}$ & $\begin{array}{l}0.23 \\
0.27 \\
0.25 \\
0.26\end{array}$ \\
\hline
\end{tabular}

${ }^{\mathrm{a}}$ Experiment reported by Tanino et al. [23].

${ }^{b}$ Experiments by Znaien et al. [22].

number

$$
\mathrm{Ri}=2 g \operatorname{At} d\left(\left.\frac{\mathrm{d}\langle\bar{u}\rangle_{x}}{\mathrm{~d} \widetilde{z}}\right|_{z=0}\right)^{-2}
$$

where $g$ is the gravitational acceleration and $\mathrm{d}\langle\bar{u}\rangle_{x} /\left.\mathrm{d} \widetilde{z}\right|_{z=0}$ is approximated by the gradient of the line of best fit over $\widetilde{z}^{-} / 2<\widetilde{z}<\widetilde{z}^{+} / 2$ ranged from $\mathrm{Ri}=0.26$ to 1.59 .

\section{Experimental results and discussion}

\subsection{Mean flow}

The mean streamwise velocity, $\langle\bar{u}\rangle_{x}$, displays a canonical dependence on $\widetilde{z}$ in all experiments considered (Figure 4, left column). Specifically, $\langle\bar{u}\rangle_{x}$ is antisymmetric about $\widetilde{z}=0$, increasing (or decreasing) almost linearly with increasing (or decreasing) $\widetilde{z}$ to an intermediate $\widetilde{z}=\widetilde{z}^{+}$(or $\widetilde{z}^{-}$), beyond which the velocity decays to $\langle\bar{u}\rangle_{x}=0$ to satisfy the 

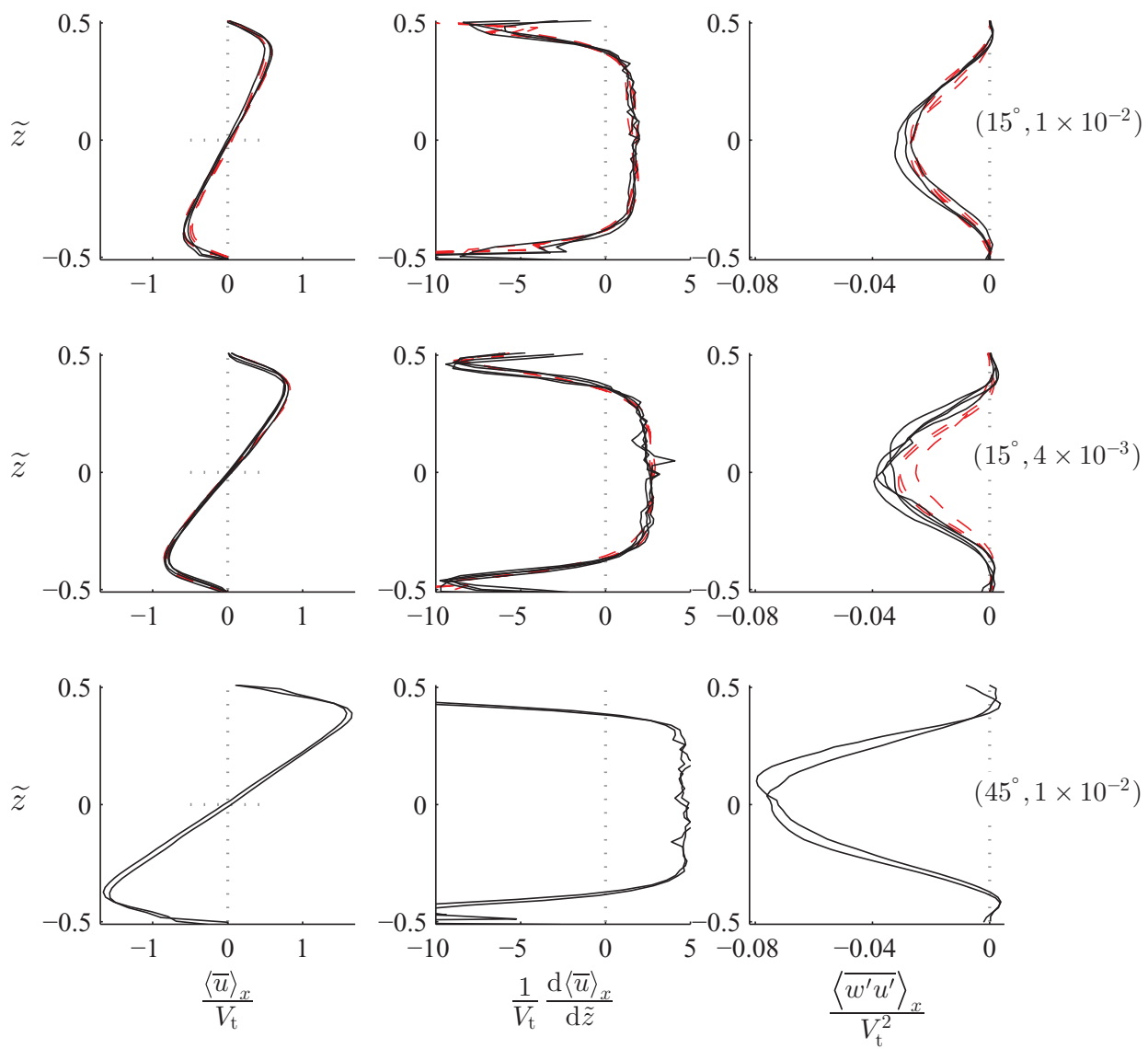

Figure 4. $\langle\bar{u}\rangle_{x} / V_{\mathrm{t}}, \mathrm{d}\langle\bar{u}\rangle_{x} / \mathrm{d} \widetilde{z} / V_{\mathrm{t}}$, and $\left\langle\overline{w^{\prime} u^{\prime}}\right\rangle_{x} / V_{\mathrm{t}}^{2}$ as a function of transverse distance from the pipe axis at each $(\theta, \mathrm{At})$. Different lines within each subplot correspond to different experimental runs at the same $(\theta, \mathrm{At}) . \mathrm{d}\langle\bar{u}\rangle_{x} / \mathrm{d} \widetilde{z}$ is estimated by a two-point finite difference. Superposed are measurements by Znaien et al. [22] (dashed).

no-slip boundary condition at the pipe wall. The maximum and minimum $\langle\bar{u}\rangle_{x}$ occur at $\widetilde{z}^{*} \equiv\left(\widetilde{z}^{+}-\widetilde{z}^{-}\right) / 2=0.37$ to 0.39 at all $(\theta, \mathrm{At})$, consistent with previous observations.[22]

Like $\langle\bar{u}\rangle_{x},\langle\bar{c}\rangle_{x}$ is broadly antisymmetric about $\widetilde{z}=0$, with $\langle\bar{c}\rangle_{x}$ increasing with increasing $\widetilde{z}$ (Figure 5, left column) and $\langle\bar{c}\rangle_{x}=0$ at the pipe axis. Consistent with previous experiments,[16] the variability over replicate runs increases rapidly with increasing $|\widetilde{z}|$ at $|\widetilde{z}| \gtrsim 0.25$. Nevertheless, an empirical function of the form

$$
\beta \frac{\langle\bar{c}\rangle_{x}}{C_{1}-C_{2}}=\tanh ^{-1}\{2 \widetilde{z}\}
$$

with $\beta$ determined by fitting Equation (7) to $\langle\bar{c}\rangle_{x}$ in the range $|\tilde{z}|<0.2$, captures $\langle\bar{c}\rangle_{x}$ reasonably well over a transverse region ranging from $0.50 d$ to $0.74 d$ in thickness (e.g., Figure 6).

The corresponding transverse gradients $\mathrm{d}\langle\bar{u}\rangle_{x} / \mathrm{d} \widetilde{z}$ and $\mathrm{d}\langle\bar{c}\rangle_{x} / \mathrm{d} \widetilde{z}$ are both symmetric with respect to $z=0$ and, in the central region of the pipe, positive and only weakly dependent on $|\widetilde{z}|$ (Figures 4 and 5, middle column). The rapid increase in $\mathrm{d}\langle\bar{c}\rangle_{x} / \mathrm{d} \widetilde{z}$ near the wall has been observed previously in both DNS [16] and laboratory experiments,[22] and 

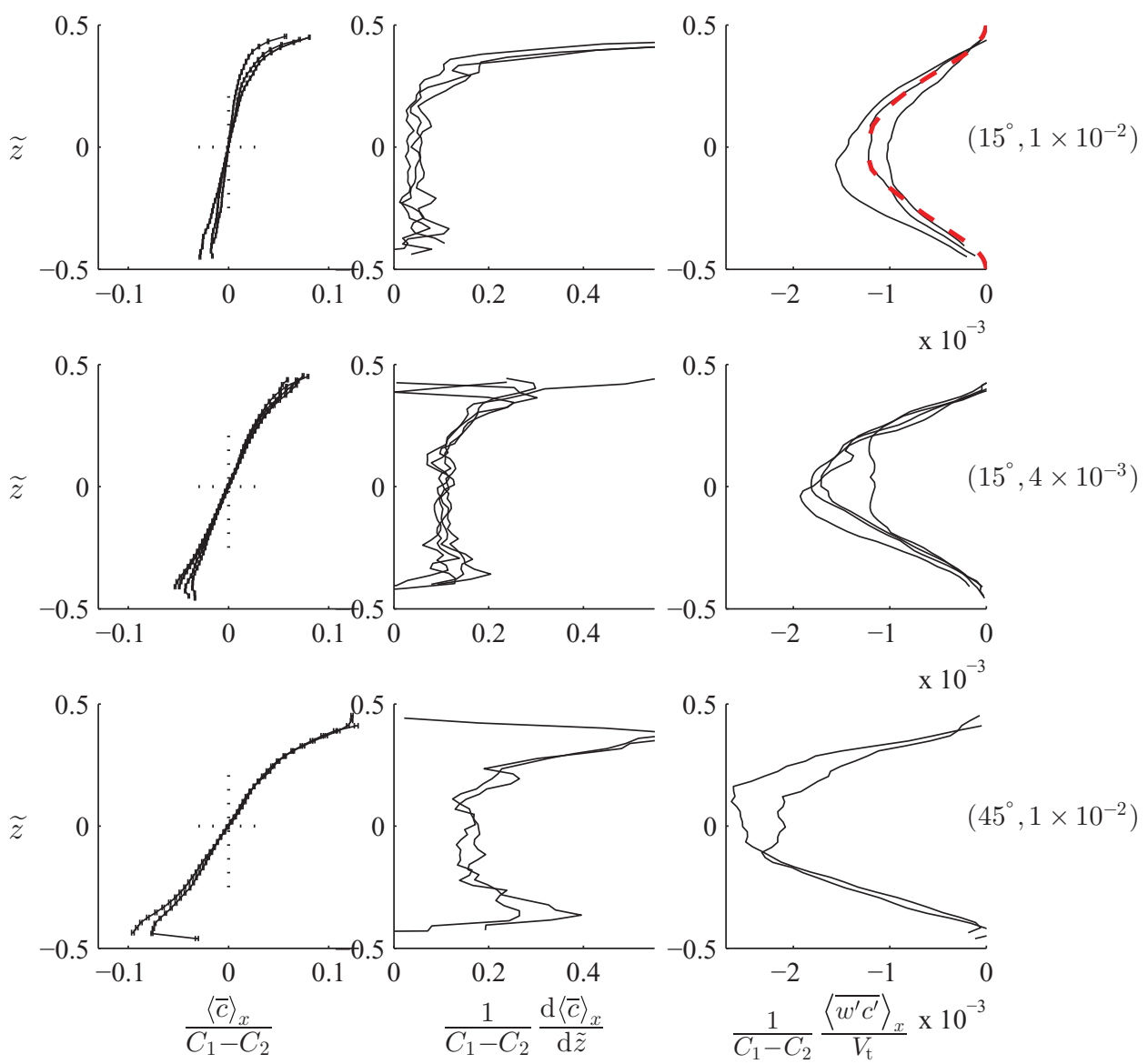

Figure 5. Transverse profiles of $\langle\bar{c}\rangle_{x}, \mathrm{~d}\langle\bar{c}\rangle_{x} / \mathrm{d} \widetilde{z}$, and $\left\langle\overline{w^{\prime} c^{\prime}}\right\rangle_{x}$. Different lines within each subplot correspond to different experimental runs at the same $(\theta, \mathrm{At}) . \mathrm{d}\langle\bar{c}\rangle_{x} / \mathrm{d} z$ is estimated by a two-point finite difference. The standard error of $\langle c\rangle_{x}$ is displayed as horizontal bars in $\langle\bar{c}\rangle_{x}$ (left column), which are of the order of the line thickness. Superposed is $\left\langle\overline{w^{\prime} c^{\prime}}\right\rangle_{x}$ predicted by DNS (unpublished data from numerical simulations by Hallez [16]; $\theta, \mathrm{At})=\left(15^{\circ}, 1.15 \times 10^{-2}\right)$; dashed $)$, which has been scaled to match the ensemble average $\left\langle\overline{w^{\prime} c^{\prime}}\right\rangle_{x}(0) /\left(C_{1}-C_{2}\right) / V_{\mathrm{t}}$ at the pipe axis in the present experiments at $(\theta, \mathrm{At})=\left(15^{\circ}, 1 \times 10^{-2}\right)$.

suggests the presence of a thin layer of relatively pure fluid along the wall.[33] Note that $\mathrm{d}\langle\bar{c}\rangle_{x} / \mathrm{d} \widetilde{z}$ in Figure 5, which are two-point finite differences, display fluctuations that are not significantly smaller than the ensemble average $\mathrm{d}\langle\bar{c}\rangle_{x} /\left.\mathrm{d} \widetilde{z}\right|_{z=0}$. These large fluctuations highlight the need to evaluate $\mathrm{d}\langle\bar{c}\rangle_{x} / \mathrm{d} \widetilde{z}$ using an empirical function such as Equation (7) when calculating $\epsilon_{c}$ and $l_{c}$.

\subsection{Eddy diffusivities}

We now consider the turbulent fluxes. Both $\left\langle\overline{w^{\prime} u^{\prime}}\right\rangle_{x}$ and $\left\langle\overline{w^{\prime} c^{\prime}}\right\rangle_{x}$ are symmetric and negative in the central region of the pipe, with the maxima of $\left|\left\langle\overline{w^{\prime} u^{\prime}}\right\rangle_{x}\right|$ and $\left|\left\langle\overline{w^{\prime} c^{\prime}}\right\rangle_{x}\right|$ occurring at $\widetilde{z}=0$ (Figures 4 and 5, right column). $-\left\langle\overline{w^{\prime} u^{\prime}}\right\rangle_{x}$ decreases as $|\widetilde{z}|$ increases until it becomes slightly negative at an intermediate distance $|\widetilde{z}|=\widetilde{z}_{\mathrm{m} 0}\left(>\widetilde{z}^{*}\right)$ near the wall, before decaying to $\left\langle\overline{w^{\prime} u^{\prime}}\right\rangle_{x}=0$ at the wall. Given the similar reversal of the mean velocity gradient at $|\widetilde{z}|=\widetilde{z}^{*}$ 
(a) $\left(15^{\circ}, 3.89 \times 10^{-3}\right)$

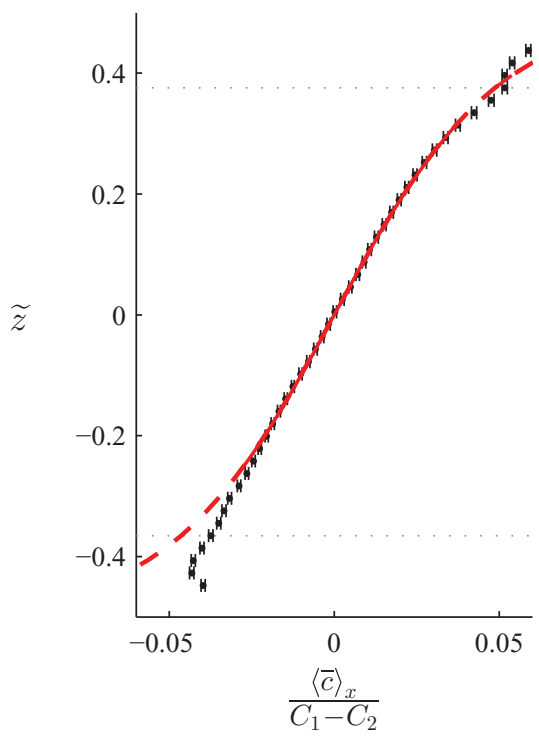

(b) $\left(45^{\circ}, 1.19 \times 10^{-2}\right)$

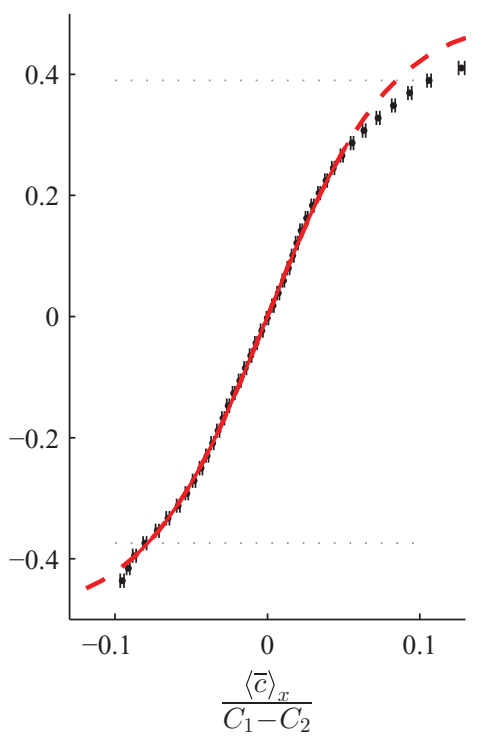

Figure 6. Transverse profiles of $\langle\bar{c}\rangle_{x} /\left(C_{1}-C_{2}\right)$ in selected runs. Black dots are measured values; the standard error over the temporal averaging window, depicted by horizontal bars, is smaller than the marker size. The solid line is Equation (7), with best fit $\beta=20.1$ (a) and 12.3 (b), for the range of $\widetilde{z}$ within which the deviation from measurement is less than $2 \%$ of $C_{1}-C_{2}$; the dashed line is its extrapolation. $\widetilde{z}=\widetilde{z}^{+}, \widetilde{z}^{-}$are also shown (grey dotted).

(Figure 4), this reversal of sign corresponds to a down-gradient turbulent flux everywhere except within the thin region $\widetilde{z}^{*}<|\widetilde{z}|<\widetilde{z}_{\mathrm{m} 0}$. In this region, both $\mathrm{d}\langle\bar{u}\rangle_{x} / \mathrm{d} z$ and $\left\langle\overline{w^{\prime} u^{\prime}}\right\rangle_{x}$ are negative.

The turbulent mass flux $-\left\langle\overline{w^{\prime} c^{\prime}}\right\rangle_{x}$ decreases as $|\widetilde{z}|$ increases, and the no-flux boundary condition imposes $\left\langle\overline{w^{\prime} c^{\prime}}\right\rangle_{x}=0$ at the pipe wall. However, unlike $\left\langle\overline{w^{\prime} u^{\prime}}\right\rangle_{x},\left\langle\overline{w^{\prime} c^{\prime}}\right\rangle_{x}$ does not display a systematic positive overshoot near the wall and, instead, decays monotonically and continuously from the pipe axis to the wall (Figure 5; [16]).

Eddy diffusivities $\epsilon_{\mathrm{m}}$ and $\epsilon_{c}$ can be determined from the present experiments by substituting the extracted values for $\left\langle\overline{w^{\prime} u^{\prime}}\right\rangle_{x}$ and $\mathrm{d}\langle\bar{u}\rangle_{x} / \mathrm{d} z$ into Equation (1a) and $\left\langle\overline{w^{\prime} c^{\prime}}\right\rangle_{x}$ and $\mathrm{d}\langle\bar{c}\rangle_{x} / \mathrm{d} z$ into Equation (1b), respectively. In calculating $\epsilon_{\mathrm{m}}$ and $\epsilon_{c}, \mathrm{~d}\langle\bar{u}\rangle_{x} / \mathrm{d} z$ was approximated by a standard two-point finite difference, while $\mathrm{d}\langle\bar{c}\rangle_{x} / \mathrm{d} z$ was taken as the derivative of Equation (7). The evaluation of $\epsilon_{c}$ was restricted to values of $\widetilde{z}$ at which the deviation of the best fit Equation (7) from the measurement was less than $2 \%$ of $C_{1}-C_{2}$. In the present experiments, this criterion precludes the analysis of $\epsilon_{c}$ (and $l_{c}$ ) at $|\widetilde{z}| \gtrsim 0.4$.

Like $\left\langle\overline{w^{\prime} u^{\prime}}\right\rangle_{x},\left\langle\overline{w^{\prime} c^{\prime}}\right\rangle_{x}, \mathrm{~d}\langle\bar{u}\rangle_{x} / \mathrm{d} \widetilde{z}$, and $\mathrm{d}\langle\bar{c}\rangle_{x} / \mathrm{d} \widetilde{z}, \epsilon_{\mathrm{m}}$ and $\epsilon_{c}$ are even functions of $\widetilde{z}$ and are thus presented as a function of $|\widetilde{z}|$ in Figure 7. The variations of $\epsilon_{\mathrm{m}}$ and $\epsilon_{c}$ with $|\widetilde{z}|$ are similar within the central region of the pipe $\left(|\widetilde{z}|<\widetilde{z}^{*}\right)$ : the maximum occurs at the pipe axis, from which the diffusivities decrease monotonically with increasing $|\widetilde{z}|$. As discussed above, we are unable to evaluate $\epsilon_{c}$ beyond this region $\left(|\widetilde{z}| \approx 0.4 \sim \widetilde{z}^{*}\right) . \epsilon_{\mathrm{m}}$, in contrast, can be evaluated in the vicinity of the wall as well. As $|\widetilde{z}| \rightarrow \widetilde{z}^{*}$, the velocity gradient vanishes while $\left\langle\overline{w^{\prime} u^{\prime}}\right\rangle_{x}$ remains finite (Figure 4) and, accordingly, $\epsilon_{\mathrm{m}} \rightarrow \infty$. In the vicinity of the wall, at $|\widetilde{z}|>\widetilde{z}_{\mathrm{m} 0}, \epsilon_{\mathrm{m}}$ is again finite and positive, and decays with increasing $|\widetilde{z}|$ until it vanishes at the wall. 
(a)

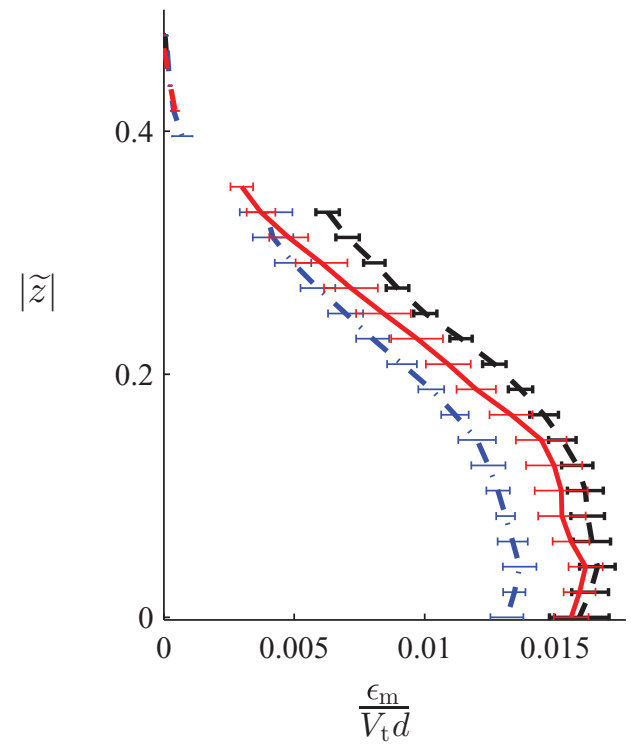

(b)

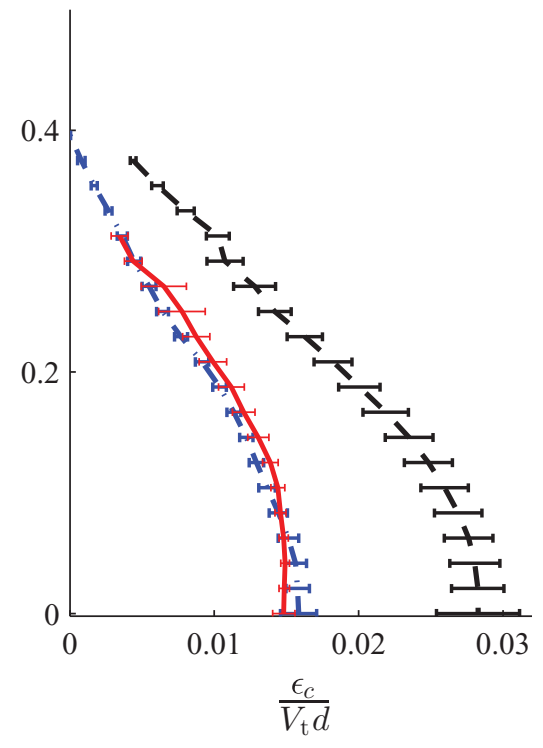

Figure 7. Ensemble average of $\epsilon_{\mathrm{m}} /\left(V_{\mathrm{t}} d\right)$ (a) and $\epsilon_{c} /\left(V_{\mathrm{t}} d\right)$ (b) at $(\theta$, At $)=\left(15^{\circ}, 1 \times 10^{-2}\right)$ (dashed), $\left(15^{\circ}, 4 \times 10^{-3}\right)$ (dashed dotted), and $\left(45^{\circ}, 1.19 \times 10^{-2}\right)$ (solid line). Horizontal bars depict the standard error over the values at $z= \pm|z|$ and over replicate runs. $\epsilon_{\mathrm{m}} /\left(V_{\mathrm{t}} d\right)$ are not presented for the region ||$\widetilde{z}\left|-\widetilde{z}^{*}\right|<\widetilde{z}_{\mathrm{m} 0}-\widetilde{z}^{*}$, where errors associated with the velocity gradient vanishing at $|\widetilde{z}|=\widetilde{z}^{*}$ are significant.

The corresponding turbulent Prandtl number, $\operatorname{Pr}_{\mathrm{t}}(|\widetilde{z}|)=\epsilon_{\mathrm{m}} / \epsilon_{c}\left(\equiv l_{\mathrm{m}} / l_{c}\right)$, is presented in Figure 8. $\mathrm{Pr}_{\mathrm{t}}$ is roughly constant in the central region of the pipe at order unity, indicating equal mixing of momentum and mass by turbulence consistent with the Reynolds analogy. The variation across different $(\theta, \mathrm{At})$ reflects a monotonic dependence on $\mathrm{Ri}$, which will be discussed in Section 3.4.

\subsection{Prandtl mixing lengths}

Assuming that turbulent mixing is dominated by the largest eddies, one would expect the Prandtl mixing lengths to scale like the integral length scale of turbulence. The Prandtl mixing lengths were evaluated using Equations (1) and (3):

$$
\widetilde{l}_{\mathrm{m}}=\sqrt{-\left\langle\overline{w^{\prime} u^{\prime}}\right\rangle_{x}\left(\frac{\mathrm{d}\langle\bar{u}\rangle_{x}}{\mathrm{~d} \widetilde{z}}\left|\frac{\mathrm{d}\langle\bar{u}\rangle_{x}}{\mathrm{~d} \widetilde{z}}\right|\right)^{-1}}
$$

and

$$
\tilde{l}_{c}=\frac{-\left\langle\overline{w^{\prime} c^{\prime}}\right\rangle_{x}}{\sqrt{-\left\langle\overline{w^{\prime} u^{\prime}}\right\rangle_{x}}}\left(\frac{\mathrm{d}\langle\bar{c}\rangle_{x}}{\mathrm{~d} \widetilde{z}}\right)^{-1}
$$




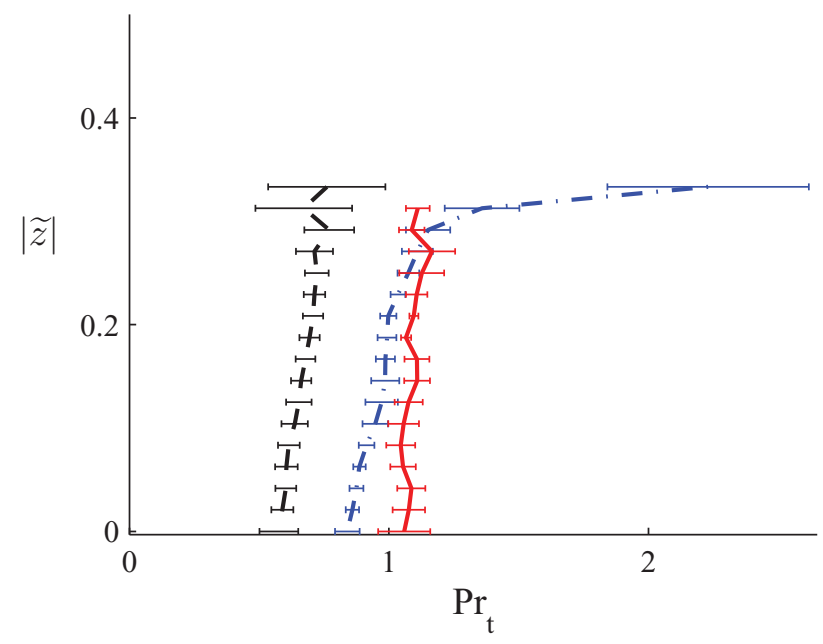

Figure 8. $\quad \operatorname{Pr}_{\mathrm{t}}$ at $(\theta, \mathrm{At})=\left(15^{\circ}, 1 \times 10^{-2}\right)($ dashed $),\left(15^{\circ}, 4 \times 10^{-3}\right)$ (dashed dotted $)$, and $\left(45^{\circ}\right.$, $\left.1.19 \times 10^{-2}\right)$ (solid line). Horizontal bars depict the standard error between $\widetilde{z}= \pm|\widetilde{z}|$ and over replicate runs.

The integral length scale was calculated at each $z$ as

$$
l_{w}(z)=\int_{0}^{r_{0}(z)} R_{3,3} \mathrm{~d} r
$$

where

$$
R_{3,3}(r, z)=\frac{\overline{\left\langle w^{\prime \prime}(x) w^{\prime \prime}(x+r)\right\rangle_{x}}}{\left\langle w^{\prime 2}\right\rangle_{x}}
$$

is the temporal average of the autocorrelation function of the transverse velocity $w(x)$ within the imaging region, $r$ is the displacement with respect to $x$, and $w^{\prime \prime}(x, z, t)=w(x, z, t)$ $-\langle w\rangle_{x}(z, t)$. We consider the correlation of $w(x)$ rather than $u(x)$ because the transverse component of velocity is more representative of local turbulence (e.g., [22,34]). While the usual definition of the integral length scale is such that $r_{0}=\infty$ in Equation (10), an alternative definition is necessary because any finite data-set would constrain $r$ to finite values and because of the uncertainty of the decay to zero of the autocorrelation functions in the present data (e.g., Figure 9). Instead, $r_{0}$ is defined as the value of $r$ at the first zero-crossing which, in general, is easily identified.

$\widetilde{l}_{w}$ decreases continuously and monotonically from its maximum at $\widetilde{z}=0$ towards the wall, with no discontinuity at $|\widetilde{z}|=\widetilde{z}^{*}$ or $\widetilde{z}_{\mathrm{m} 0}$ (Figure 10 , left column). While $l_{\mathrm{m}}$ and $l_{c}$ display a qualitatively similar dependence on $\widetilde{z}$ within the central $60 \%$ of the pipe cross-section (middle column), $l_{\mathrm{m}} / l_{w}$ and $l_{c} / l_{w}$ retain some $|\tilde{z}|$ dependence (right).

The Reynolds analogy implies that the two normalised mixing lengths, $l_{\mathrm{m}} / l_{w}$ and $l_{c} / l_{w}$, are approximately the same and are independent of $(\theta$, At). While this is indeed the case at $(\theta$, At $)=\left(15^{\circ}, 4 \times 10^{-3}\right)$ and $\left(45^{\circ}, 1 \times 10^{-2}\right)$, this does not extend to $(\theta, \mathrm{At})=\left(15^{\circ}\right.$, $\left.1 \times 10^{-2}\right)$, where both $l_{\mathrm{m}}$ and $l_{c}$ are larger than in the previous cases and, moreover, $l_{c}$ is up to $70 \%$ larger than $l_{\mathrm{m}}$. The difference between $l_{\mathrm{m}} / l_{w}$ and $l_{c} / l_{w}$ and between different 

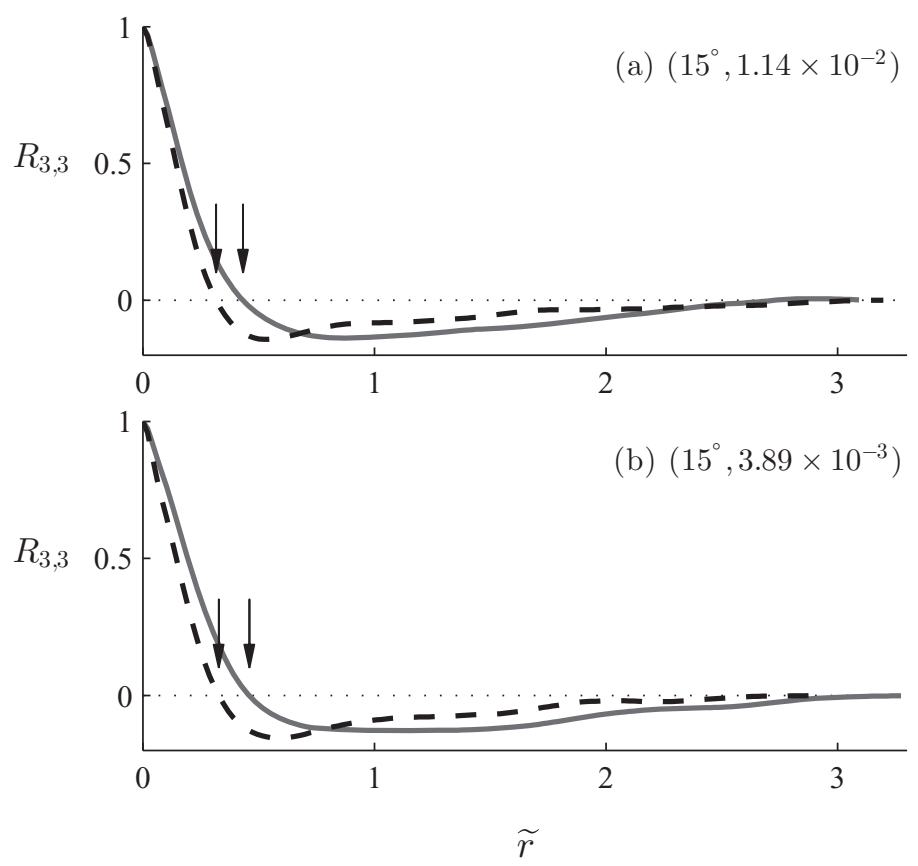

Figure 9. The temporal average of the normalised autocorrelation function at $|\widetilde{z}| \approx 0$ (solid) and 0.3 (dashed) in two runs: J19 (a) and J07 (b). Arrows mark $r_{0}$.

$(\theta, \mathrm{At})$ arise from the dependence of the mixing lengths on $\mathrm{Ri}$, which is explored further in Section 3.4.

\subsection{Dependence on $\operatorname{Re}$ and $\mathrm{Ri}$}

The behaviour of turbulent stratified shear flows are generally governed by Re and Ri. In the following, we consider the dependence of $\operatorname{Pr}_{\mathrm{t}}, \widetilde{l}_{\mathrm{m}}, \widetilde{l}_{c}$, and $\widetilde{l}_{w}$ on these two parameters separately. For simplicity, we focus on the values of these parameters at the pipe axis, which correspond to their local maximum.

$\widetilde{l}_{w}(0), \widetilde{l}_{\mathrm{m}}(0)$, and $\widetilde{l}_{c}(0)$, and hence $\operatorname{Pr}_{\mathrm{t}}(0) \equiv l_{\mathrm{m}}(0) / l_{c}(0)$, do not vary systematically with $\operatorname{Re}$ (Figure 11), consistent with previous observations of a very weak Re dependence of $\mathrm{Pr}_{t}$ in uniform pipe flow [35,36] and in stratified air flows [37] at $\operatorname{Re} \geq 10^{4}$. Combined with the constant shear layer thickness of $\widetilde{z}^{*}=0.38$ and the similarity of the mean velocity profile observed across all experiments (Section 3.1), these results are consistent with fully turbulent conditions.

Next, we consider the dependence of the same parameters on Ri. $\widetilde{l}_{w}(0)$ [•, Figure 12(b)] is independent of $\mathrm{Ri}$, which is consistent with the expectation that the integral length scale in a shear layer scales with the thickness of that layer which, in this study, is constant $\left(=2 \widetilde{z}^{*} \approx 0.76\right)$ across all experiments. $\widetilde{l}_{\mathrm{m}}(0)$ and $\widetilde{l}_{c}(0)$, in contrast, display a strong, positive, linear correlation with Ri (solid lines), Figure 12(b). A stronger stratification is thus correlated with longer mixing lengths and, since $\widetilde{l}_{w}(0)$ is constant, with larger values of $l_{\mathrm{m}}(0) / l_{w}(0)$ and $l_{c}(0) / l_{w}(0)$. Combined, $\operatorname{Pr}_{\mathrm{t}}(0)$ decreases with increasing $\mathrm{Ri}$, with $\operatorname{Pr}_{\mathrm{t}}(0)=1$ at $\mathrm{Ri}=0.33$ (Figure 12(a)). This Ri dependence is consistent with the negative correlation between $\mathrm{Pr}_{t}$ and bulk Richardson number reported in turbulent thermally stratified shear 
(a) $\left(15^{\circ}, 1 \times 10^{-2}\right)$
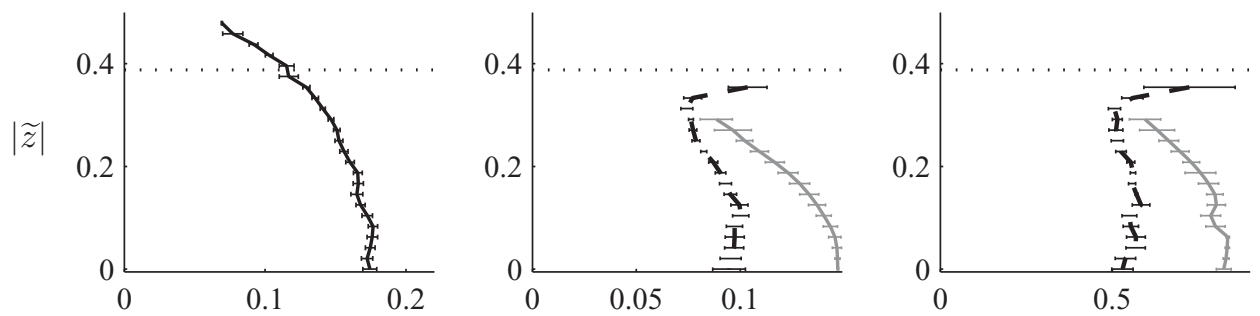

(b) $\left(15^{\circ}, 4 \times 10^{-3}\right)$
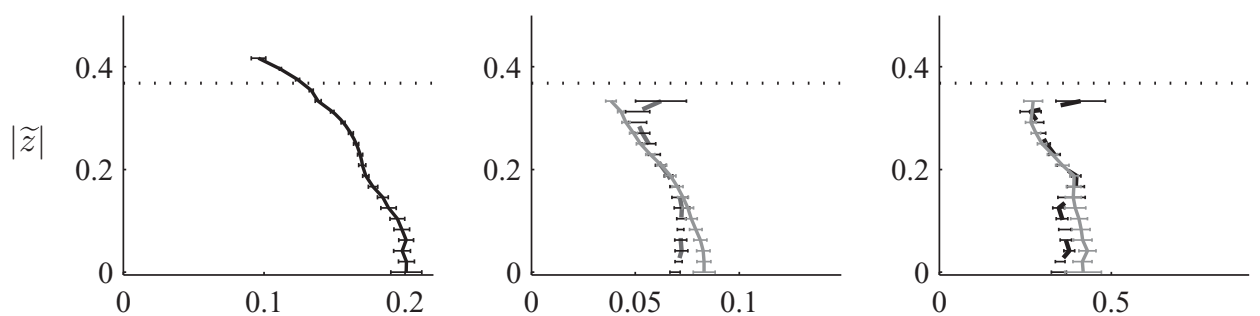

(c) $\left(45^{\circ}, 1 \times 10^{-2}\right)$
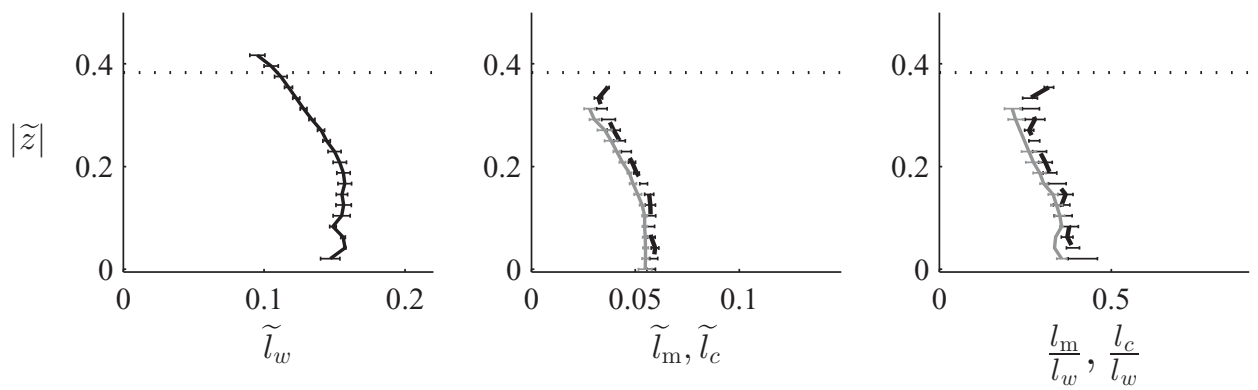

Figure 10. Ensemble average of $\tilde{l}_{w}$ (left column), $\tilde{l}_{\mathrm{m}}$ (middle column, dashed line), and $\tilde{l}_{c}$ (middle, grey solid line), and $l_{\mathrm{m}} / l_{w}$ (right column, dashed), and $l_{c} / l_{w}$ (right, grey solid) at each $(\theta$, At). Because $\mathrm{At}$, and hence Ri, in run J14 deviate from those in the other two runs at $\theta$, At $)=\left(15^{\circ}, 1 \times 10^{-2}\right)$ by $20 \%$, run J14 is not considered in the ensemble average of $\widetilde{l}_{\mathrm{m}}$ and $\widetilde{l}_{c}$ (cf. Section 3.4). Horizontal bars depict the standard error of the mean over replicate runs and between $z= \pm|z|$, where they are not visible they are smaller than the thickness of the lines. Ensemble average $|\widetilde{z}|=\widetilde{z}^{*}$ are also shown (dotted).

flows, both in the stable atmospheric boundary layer [38,39] and in a wind tunnel,[39] and implies that there is no universal value of $\operatorname{Pr}_{\mathrm{t}}, \widetilde{l}_{\mathrm{m}}$, or $\widetilde{l}_{c}$.

At first glance, a positive correlation between $\widetilde{l}_{\mathrm{m}}-$ a measure of turbulence intensity (Equation (3)) - and stratification may seem counter-intuitive. However, in flows considered presently, the density contrast is associated with both stronger stratification and larger $\Delta U$. The former tends to suppress turbulent mixing and reduce $l_{\mathrm{m}}$, but the latter tends to promote turbulence and enhance $l_{\mathrm{m}}$. Furthermore, negative feedback exists between the two processes: weaker turbulence sustains the density contrast at the propagating front that drives the mean flow, which in turn results in a larger $\Delta U$. The observed Ri dependence may be attributed to the subtle competition between these effects. 


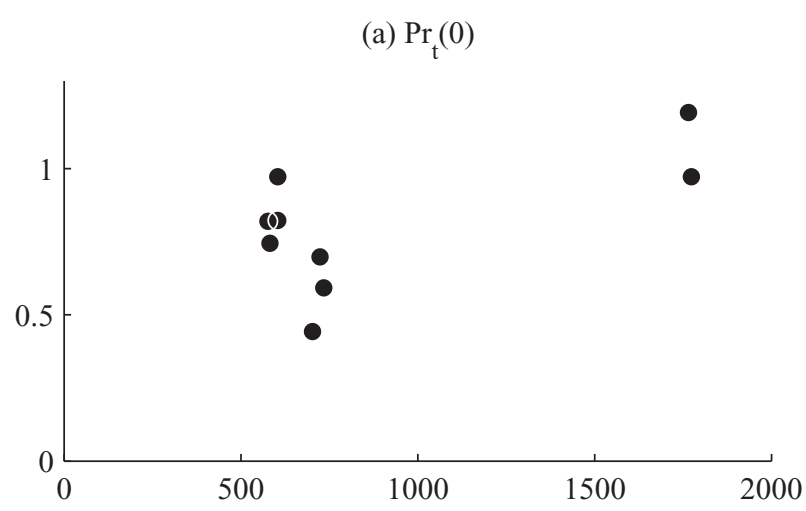

(b)

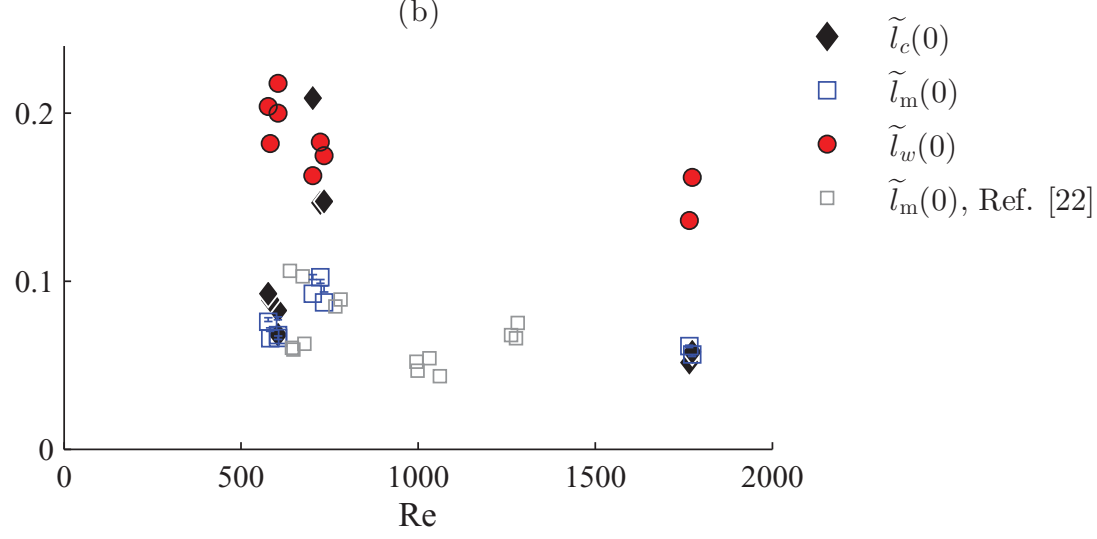

Figure 11. (a) $\operatorname{Pr}_{\mathrm{t}}(z=0)$ and $(\mathrm{b}) \widetilde{l}_{\mathrm{m}}(0), \widetilde{l}_{c}(0)$, and $\widetilde{l}_{w}(0)$ as a function of $\operatorname{Re}$ for all $(\theta, \mathrm{At}) \cdot \widetilde{l}_{\mathrm{m}}(0)$ reported by Znaien et al. [22] is also presented.

\subsection{Comparison with other classes of shear flows}

We now compare the integral length scale and Prandtl mixing lengths in flows considered presently with those in other classes of shear flows. Specifically, we consider plane mixing layers, open-channel flows through submerged cylinder arrays ('canopy flows'), and a buoyant jet released along an inclined plane.

To facilitate comparison with unbounded and semi-bound flows, we normalise the lengths by the shear layer thickness,

$$
L_{\mathrm{s}}=\frac{\Delta U}{\mathrm{~d}\langle\bar{u}\rangle_{x} /\left.\mathrm{d} z\right|_{z=0}},
$$

where $z=0$ is defined at the transverse position of maximum shear. The normalised mixing lengths, like $l_{\mathrm{m}}(0)$ and $l_{c}(0)$, were Ri dependent in the present experiments. Their extrapolation to $\mathrm{Ri}=0$ yields $\left(l_{\mathrm{m}}(0) / L_{\mathrm{s}}, l_{c}(0) / L_{\mathrm{s}}\right)=(0.079 \pm 0.006,0.03 \pm 0.02)$, which are $20 \%$ and $70 \%$ smaller than mixing lengths reported in unstratified canopy flows $\left[l_{\mathrm{m}}(0) / L_{\mathrm{s}}=\right.$ $0.10 \pm 0.01]$.[15] $\left(l_{\mathrm{m}}(0) / L_{\mathrm{s}}, l_{c}(0) / L_{\mathrm{s}}\right)$ in flows considered presently are also smaller, although only by $8 \%$ and $20 \%$, respectively, than those in a buoyant jet bound on one side by an inclined plane considered by Odier et al. [40]. ${ }^{2}$ 
(a) $\operatorname{Pr}_{t}(0)$

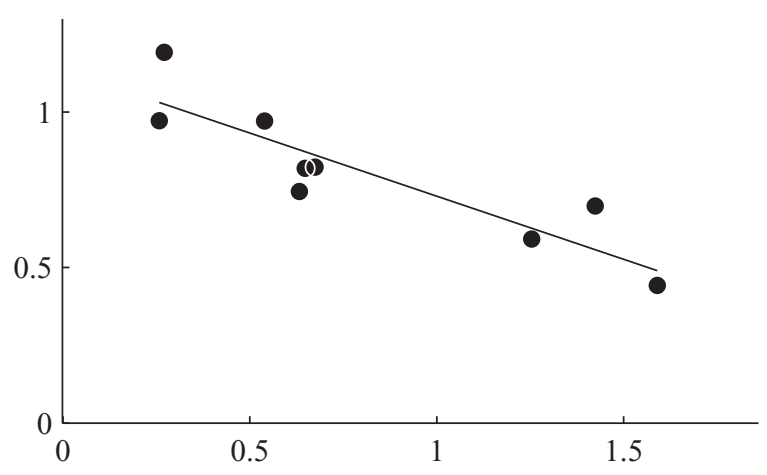

(b)

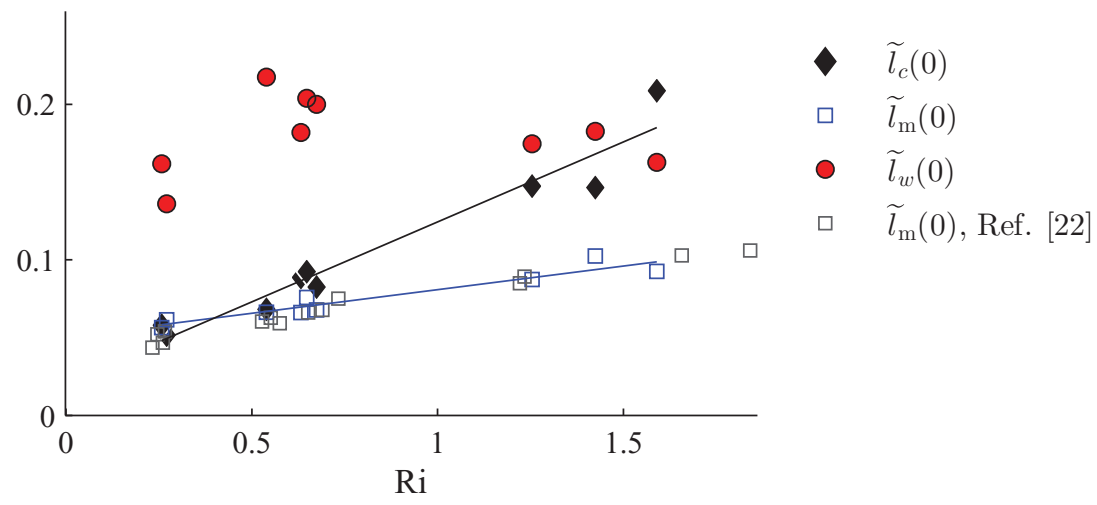

Figure 12. Data in Figure 11 plotted as a function of Ri; see caption for Figure 11 for marker definition. Solid lines are the lines of best fit in the least squares sense.

Similarly, $l_{w}(0) / L_{\mathrm{s}}=0.28 \pm 0.05$ measured in this study are smaller than in conventional (i.e., unstratified and unbounded) plane mixing layers, in which $l_{w}(0) / L_{\mathrm{s}}=0.6$ to 0.8 typically,[41] as well as $l_{w}(0) / L_{\mathrm{s}}=0.8$ to 1.5 in canopy flows.[42] Combined, these relative magnitudes suggest that strong confinement tends to reduce the integral length and Prandtl mixing lengths in addition to restricting the shear layer thickness itself.

\section{Conclusions}

In a lock-exchange flow in an inclined pipe under uniformly turbulent conditions, both the turbulent transverse fluxes and the mean transverse gradients of mass and momentum are symmetric about the pipe axis. The mean concentration gradient, $\mathrm{d}\langle\bar{c}\rangle_{x} / \mathrm{d} \widetilde{z}$, is everywhere positive, and the mean velocity gradient, $\mathrm{d}\langle\bar{u}\rangle_{x} / \mathrm{d} \widetilde{z}$, is positive in the central region of the pipe $\left(|\widetilde{z}|=\widetilde{z}^{*} \lesssim 0.38\right)$ and negative in the wall boundary layer. The magnitude of the fluxes are largest at the axis, where shear is highest, and decay smoothly away from the pipe axis towards the wall. The corresponding turbulent eddy diffusivities, as well as the integral length scale of turbulence, are strong functions of $|\widetilde{z}|$. The corresponding Prandtl mixing 
lengths also decrease away from the pipe axis, albeit more weakly. Consequently, neither the constant diffusivity nor the (constant) mixing length model apply fully to present data.

The mixing lengths at the pipe axis increase linearly with $\mathrm{Ri}$, with the mixing length of mass increasing more rapidly than that of momentum. Accordingly, the corresponding Prandtl number decreases with increasing Ri. In contrast, these variables do not vary monotonically with Re. The dependence on Ri is interesting, given that the onset of instability is controlled by Re and not by the local Richardson number.[23]

The magnitudes of the mixing lengths relative to the thickness of the shear layer in the centre of the pipe are smaller in flows considered presently than in a buoyant jet bound on one side by a wall and in open-channel canopy flows. Similarly, the integral length scale normalised by the shear layer thickness is smaller in this study than in canopy flows and in unbounded plane mixing layers. These comparison suggest that the strong confinement imposed by the pipe wall in the present configuration constrains the integral length scale and mixing lengths. Additional measurements in pipes of different diameters are necessary to test this hypothesis.

\section{Acknowledgements}

Frédéric Moisy acknowledges the Institut Universitaire de France for its support. The authors thank Jemil Znaien for providing unpublished details of data reported in Znaien et al. [22], Yannick Hallez for providing unpublished data from Znaien et al. [16], and Christian Borget and Rafael Pidoux for the construction of the experimental set-up. We also thank the two anonymous reviewers for their insightful comments.

\section{Disclosure statement}

No potential conflict of interest was reported by the authors.

\section{Funding}

This material is based on work supported by the Agence Nationale pour la Recherche [grant ANR07-BLAN-0181].

\section{Notes}

1. Note that this definition differs from the one used in Ref. [21] by a factor $\sqrt{\cos \theta / 2}$.

2. Note that our definition of Richardson number and Prandtl mixing length for mass differ from those used in Ref. [40]. Ri and $\widetilde{l}_{c}(0)$ for Odier et al. [40]'s experiments were calculated as defined by Equation (6) and by $\widetilde{l}_{c}=-\left\langle\overline{w^{\prime} \rho^{\prime}}\right\rangle_{x}\left(\langle\overline{\mathrm{d} \rho / \mathrm{d} \widetilde{z}}\rangle_{x}\right)^{-1}\left(-\left\langle\overline{w^{\prime} u^{\prime}}\right\rangle_{x}\right)^{-1 / 2}$, respectively, taking $d$ (= $8 \mathrm{~cm})$ to be equal to twice the distance between the depth of maximum $\left|\left\langle\overline{w^{\prime} u^{\prime}}\right\rangle\right|$ and the upper boundary and $\mathrm{d}\langle\bar{u}\rangle_{x} /\left.\mathrm{d} z\right|_{0}=-1.4 \mathrm{~s}^{-1}$ as reported in Ref. [40].

\section{ORCID}

Yukie Tanino 미 http://orcid.org/0000-0002-5766-0515

\section{References}

[1] Brice JW Jr, Holmes BC. Engineered casing cementing programs using turbulent flow techniques. J Petroleum Technol. 1964;16:503-508.

[2] Kamath VA, Godbole SP. Evaluation of hot-brine stimulation technique for gas production from natural gas hydrates. J Petroleum Technol. 1987;39:1379-1388. 
[3] Saurel C, Petersen JK, Wiles PJ, Kaiser MJ. Turbulent mixing limits mussel feeding: direct estimates of feeding rate and vertical diffusivity. Marine Ecol Prog Ser. 2013;485:105-121.

[4] Cloern JE. Does the benthos control phytoplankton biomass in South San Francisco Bay? Marine Ecol Prog Ser. 1982;9:191-202.

[5] Eckman JE, Okamura B. A model of particle capture by bryozoans in turbulent flow: significance of colony form. Am Naturalist. 1998;152:861-880.

[6] Velasco E, Pressley S, Grivicke R, Allwine E, Coons T, Foster W, Jobson T, Westberg H, Ramos R, Hernandez F, Molina LT, Lamb B. Eddy covariance flux measurements of pollutant gases in urban Mexico City. Atmospheric Chem Phys Discuss. 2009;9:7991-8034.

[7] Raupach MR, Thom AS. Turbulence in and above plant canopies. Annu Rev Fluid Mech. 1981;13:97-129.

[8] Kondo J, Kanechika O, Yasuda N. Heat and momentum transfers under strong stability in the atmospheric surface layer. J Atmospheric Sci. 1978;35:1012-1021.

[9] Bonan GB. Forests and climate change: forcings, feedbacks, and the climate benefits of forests. Science. 2008;320:1444-1449.

[10] Bala G, Caldeira K, Wickett M, Phillips TJ, Lobell DB, Delire C, Mirin A. Combined climate and carbon-cycle effects of large-scale deforestation. Proc Natl Acad Sci USA. 2007;104:65506555 .

[11] Reidenbach MA, Monismith SG, Koseff JR, Yahel G, Genin A. Boundary layer turbulence and flow structure over a fringing coral reef. Limnology Oceanography. 2006;51:1956-1968.

[12] Crimaldi JP, Koseff JR, Monismith SG. A mixing-length formulation for the turbulent Prandtl number in wall-bounded flows with bed roughness and elevated scalar sources. Phys Fluids. 2006;18:095102.

[13] Coceal O, Belcher SE. A canopy model of mean winds through urban areas. Q J Royal Meteorol Soc. 2004;130:1349-1372.

[14] Tsujimoto T, Shimizu Y, Kitamura T, Okada T. Turbulent open-channel flow over bed covered by rigid vegetation. J Hydrosci Hydraulic Eng. 1992;10:13-25.

[15] Ghisalberti M, Nepf HM. The limited growth of vegetated shear layers. Water Resour Res. 2004;40:W07502.

[16] Znaien J, Hallez Y, Moisy F, Magnaudet J, Hulin JP, Salin D, Hinch EJ. Experimental and numerical investigations of flow structure and momentum transport in a turbulent buoyancydriven flow inside a tilted tube. Phys Fluids. 2009;21:115102.

[17] Odier P, Chen J, Rivera MK, Ecke RE. Fluid mixing in stratified gravity currents: the Prandtl mixing length. Phys Rev Lett. 2009;102:134504.

[18] Xu D, Chen J. Experimental study of stratified jet by simultaneous measurements of velocity and density fields. Exp Fluids. 2012;53:145-162.

[19] Cossu R, Wells MG. A comparison of the shear stress distribution in the bottom boundary layer of experimental density and turbidity currents. Eur J Mech. 2012;32:70-79.

[20] Corrsin S. Limitations of gradient transport models in random walks and in turbulence. Adv Geophys. 1974;18A:25-60.

[21] Séon T, Znaien J, Salin D, Hulin JP, Hinch EJ, Perrin B. Transient buoyancy-driven front dynamics in nearly horizontal tubes. Phys Fluids. 2007;19:123603.

[22] Znaien J, Moisy F, Hulin JP. Flow structure and momentum transport for buoyancy driven mixing flows in long tubes at different tilt angles. Phys Fluids. 2011;23:035105.

[23] Tanino Y, Moisy F, Hulin JP. Laminar-turbulent cycles in inclined lock-exchange flows. Phys Rev E. 2012;85:066308.

[24] Kasarova SN, Sultanova NG, Ivanov CD, Nikolov ID. Analysis of the dispersion of optical plastic materials. Opt Mater. 2007;29:1481-1490.

[25] Daimon M, Masumura A. Measurement of the refractive index of distilled water from the near-infrared region to the ultraviolet region. Appl Opt. 2007;46:3811-3820.

[26] Séon T, Hulin JP, Salin D, Perrin B, Hinch EJ. Laser-induced fluorescence measurements of buoyancy driven mixing in tilted tubes. Phys Fluids. 2006;18:041701/1-4.

[27] Séon T, Hulin JP, Salin D, Perrin B, Hinch EJ. Buoyant mixing of miscible fluids in tilted tubes. Phys Fluids. 2004;16.

[28] Séon T. Du mélange turbulent aux courants de gravité en géométrie confinée [dissertation]. Orsay (France): University of Paris; 2006.

[29] Séon T, Znaien J, Perrin B, Hinch EJ, Salin D, Hulin JP. Front dynamics and macroscopic diffusion in buoyant mixing in a tilted tube. Phys Fluids. 2007;19:125105/1-7. 
[30] Schwarzenbach RP, Gschwend PM, Imboden DM. Environmental organic chemistry. 2nd ed. Hoboken (NJ): John Wiley \& Sons, Inc.; 2003.

[31] Haynes WM. Concentrative properties of aqueous solutions: density, refractive index, freezing point depression, and viscosity. In: Haynes WM, editor. CRC handbook of chemistry and physics. 91st ed. [Internet version]. Boca Raton (FL): CRC Press; 2011.

[32] Kestin J, Sokolov M, Wakeham WA. Viscosity of liquid water in the range $-8^{\circ} \mathrm{C}$ to $150^{\circ}$. $\mathrm{J}$ Phys Chem Ref Data. 1978;7:941-948.

[33] Hallez Y, Magnaudet J. Effects of channel geometry on buoyancy-driven mixing. Phys Fluids. 2008;20:1-9.

[34] Finnigan J. Turbulence in plant canopies. Annu Rev Fluid Mech. 2000;32:519-571.

[35] Groenhof HC. Eddy diffusion in the central region of turbulent flows in pipes and between parallel plates. Chem Eng Sci. 1970;25:1005-1014.

[36] Sheriff N, O'Kane DJ. Eddy diffusivity of mass measurements for air in circular duct. Int J Heat Mass Transfer. 1971;14:697-707.

[37] Chia W-S, Sage BH. Temperature gradients in turbulent gas streams: Investigation of the limiting value of total Prandtl number. AIChE J. 1970;16:37-43.

[38] Grachev AA, Andreas EL, Fairall CW, Guest PS, Persson POG. On the turbulent Prandtl number in the stable atmospheric boundary layer. Bound-Layer Meteorol. 2007;125:329-341.

[39] Chuang H, Renda RB. Turbulent Prandtl number in thermally stratified shear flows of air. Int J Heat Mass Transfer. 1969;12:1585-1594.

[40] Odier P, Chen J, Ecke RE. Understanding and modeling turbulent fluxes and entrainment in a gravity current. Phys D. 2012;241:260-268.

[41] Raupach MR, Finnigan JJ, Brunet Y. Coherent eddies and turbulence in vegetation canopies: the mixing-layer analogy. Bound-Layer Meteorol. 1996;78:351-382.

[42] Nezu I, Sanjou M. Turbulence structure and coherent motion in vegetated canopy open-channel flows. J Hydro-environ Res. 2008;2:62-90. 